\title{
Impact of land reclamation and agricultural water regime on the distribution and conservation status of the endangered Dryophytes suweonensis
}

Amaël Borzée ${ }^{\text {Corresp., }}{ }^{1,2}$ ， Kyungmin Kim ${ }^{2}$ ， Kyongman Heo ${ }^{2,3}$ ， Piotr G Jablonski ${ }^{\text {Corresp., }}{ }^{1,4}$ ， Yikweon Jang ${ }^{\text {Corresp. }}$ 2, 5

1 Laboratory of Behavioral Ecology and Evolution, School of Biological Sciences, Seoul National University, Seoul, South Korea

2 Division of EcoScience, Ewha Women's University, Seoul, South Korea

3 College of Natural Science, Sangmyung University, Seoul, South Korea

4 Museum and Institute of Zoology, Polish Academy of Sciences, Warsaw, Poland

5 Interdisciplinary Program of EcoCreative, Ewha Women's University, Seoul, South Korea

Corresponding Authors: Amaël Borzée, Piotr G Jablonski, Yikweon Jang

Email address: amaelborzee@gmail.com, piotrjab@hotmail.com, jangy@ewha.ac.kr

Knowledge about the distribution and habitat preferences of a species is critical for its conservation. The Suweon Treefrog (Dryophytes suweonensis) is an endangered species endemic to the Republic of Korea. We conducted surveys from 2014 to 2016 at 890 potentially suitable sites across the entire range of the species in South Korea. We then assessed whether $D$. suweonensis was found in the current and ancestral predicted ranges, reclaimed and protected areas, and how the presence of agricultural floodwater affected its occurrence. Our results describe a $120 \mathrm{~km}$ increase in the southernmost known distribution of the species, and the absence of the species at lower latitudes. We then demonstrate a putative constriction on the species ancestral range due to urban encroachment, and provide evidence for a significant increase in its coastal range due to the colonisation of reclaimed land by the species. In addition, we demonstrate that $D$. suweonensis is present in rice fields that are flooded with water originating from rivers as opposed to being present in rice fields that are irrigated from underground water. Finally, the non-overlap of protected areas and the occurrence of the species shows that only the edge of a single site where $D$. suweonensis occurs is legally protected. Based on our results and the literature, we suggest the design of a site fitting all the ecological requirements of the species, and suggest the use of such sites to prevent further erosion in the range of $D$. suweonensis. 
3 Amaël Borzée ${ }^{1,2}$, Kyungmin $\mathrm{Kim}^{2}$, Kyongman $\mathrm{Heo}^{2,3}$, Piotr G. Jablonski ${ }^{1,4^{*}}$ and Yikweon Jang ${ }^{2,5, *}$

$4{ }^{1}$ Laboratory of Behavioral Ecology and Evolution, School of Biological Sciences, Seoul National

5 University, Seoul, 08826, Republic of Korea.

$6{ }^{2}$ Division of EcoScience, Ewha Womans University, Seoul, 03760, Republic of Korea.

$7 \quad{ }^{3}$ College of Natural Science, Sangmyung University, Seoul 03016, Republic of Korea.

$8{ }^{4}$ Museum and Institute of Zoology, Polish Academy of Sciences, Warsaw 00-679, Poland

$9{ }^{5}$ Interdisciplinary Program of EcoCreative, Ewha Womans University, Seoul, 03760, Republic of

10 Korea.

$11 *$ Corresponding authors

12 Running head: Dryophytes suweonensis range and threats

\section{ABSTRACT}

14 Knowledge about the distribution and habitat preferences of a species is critical for its 15 conservation. The Suweon Treefrog (Dryophytes suweonensis) is an endangered species endemic 16 to the Republic of Korea. We conducted surveys from 2014 to 2016 at 890 potentially suitable 17 sites across the entire range of the species in South Korea. We then assessed whether $D$. 
18 suweonensis was found in the current and predicted ancestral ranges, reclaimed and protected

19 areas, and how the presence of agricultural floodwater affected its occurrence. Our results 20 describe a $120 \mathrm{~km}$ increase in the southernmost known distribution of the species, and the

21 absence of the species at lower latitudes. We then demonstrate a putative constriction on the

22 species ancestral range due to urban encroachment, and provide evidence for a significant

23 increase in its coastal range due to the colonisation of reclaimed land by the species. In addition,

24 we demonstrate that $D$. suweonensis is present in rice fields that are flooded with water

25 originating from rivers as opposed to being present in rice fields that are irrigated from

26 underground water. Finally, the non-overlap of protected areas and the occurrence of the species

27 shows that only the edge of a single site where D. suweonensis occurs is legally protected. Based

28 on our results and the literature, we suggest the design of a site fitting all the ecological

29 requirements of the species, and suggest the use of such sites to prevent further erosion in the 30 range of D. suweonensis.

\section{INTRODUCTION}

32 Very few species have a cosmopolitan distribution, and most are likely to be under local

33 environmental pressure (Purvis et al. 2000). When the entire range of a species is threatened by

34 urbanization or other types of habitat modification, the risk of extinction increases exponentially

35 (Huxley 2013). As a result, the assessment of extinction risks depend on threat levels (Mace \&

36 Lande 1991; see IUCN 2016), which may guide optimal conservation effort to prevent extinction

37 (Pimm et al. 2014).

39 have been easily avoided. For example, the Tecopa pupfish (Cyprinodon nevadensis calidae)

40 became extinct following construction of man-made structures on the Tecopa Hot Springs, the 
41 only site where the species occurred (Miller et al. 1989). Unfortunately, this information was not

42 available at the time of construction. Knowledge of species' habitat preferences provides

43 background information for the assessment of extinction risks (Manne \& Pimm 2001), and can be

44 used to develop spatial models for species' distribution (Corsi et al. 2000). For instance, a

45 subspecies of Ursini's viper, Vipera ursinii graeca, was known to occur only in Greece and at a

46 single locality in Albania. However, eight new localities were found through landscape and

47 climate modelling, doubling the known range of the species (Mizsei et al. 2016).

Although critical, obtaining information about species' ranges and habitat preferences is only a first step for any conservation effort. At risk species with clearly defined ranges still go extinct in large numbers and a way to stem this loss is through the implementation of protected

51 areas (Pimm et al. 2014). The occurrence of a species within a protected area will significantly

52 increase its chance of survival, despite the debated effectiveness of currently located protected 53 areas (Abellán \& Sánchez-Fernández 2015), and the need for the establishment of additional 54 protected areas (Brooks et al. 2004).

The Class Amphibia is currently the most endangered class of vertebrates (Stuart et al.

56 2004). Among the difficulties for amphibian conservation efforts are unknown distribution limits

57 and the absence of adequate breeding sites. Suitable natural wetlands for amphibians have been

58 converted into farmlands such as rice-paddies over the last century, especially in the Republic of

59 Korea (Juliano 1993; Czech \& Parsons 2002; Machado \& Maltchik 2010). Furthermore, those

60 farmlands still holding a fraction of the original biodiversity are being converted into residential

61 and commercial facilities at an alarming rate. In the Republic of Korea, rice production has

62 decreased by about $25 \%$ since peak production in the 1970s (FAO 2016). Since then, there have

63 been clear negative repercussions on habitats available for amphibians (Park et al. 2014). 
65 et al. 2016), is an endangered, endemic treefrog species from the Korean Peninsula. As of 2012,

66 the species was known to occur in a very restricted range, limited to five valleys centred in

67 metropolitan Seoul (Kim et al. 2012). It is therefore possible that the largest populations of $D$.

68 suweonensis might have been historically present in and around the present Seoul area (Borzée et

69 al. 2015a). Yet, opportunistic observations of calling males in the Democratic People's Republic

70 of Korea (Chun et al. 2012) and further south than previously reported (Borzée et al. 2016c), lead

71 to the expectation of a broader distribution for the species.

72 Dryophytes suweonensis is an evolutionary important species due to its unusual ZW

73 karyotype, warranting special conservation efforts (Dufresnes et al. 2015). Here, we first aimed to

74 describe the extent of occurrence and distribution of the species through occurrence surveys, as

75 well as the loss of ancestral range because of urbanisation. We then assessed the overlap between

76 the range of the species and reclaimed tidal flats, and the overlap between range and protected

77 areas. Next, because the distribution of $D$. suweonensis is closely intertwined with rice

78 cultivation, we examined whether the origin of agricultural flood waters was critical for the

79 occurrence of the species. Finally, we extracted environmental variables collected from field

80 surveys and described optimal conservation sites for D. suweonensis.

81 MATERIAL AND METHODS

82 Field surveys were conducted during 2014, 2015 and 2016, only after the beginning of the

83 breeding season of the species (Roh et al. 2014) to prevent any false negative. Because

84 Dryophytes suweonensis has not been observed using other vegetation than rice seedlings as

85 supports from which to hang to produce advertisement calls (Borzée et al. 2016b), and because it

86 is not known to breed in any other wetland than rice paddies (Borzée and Jang 2015), the species

87 typically starts breeding after rice planting. 
The setting of modern rice fields during the last decades led to a specific geometric

89 grouping of rice paddies, here referred to as rice-paddy complexes. A rice-paddy complex is

90 characterized by a central ditch running mostly straight through the complex for irrigation

91 purposes. Along this central ditch, and thus along the longest and straightest line available,

92 usually runs a cemented lane, typically following the centre of the valley. In this study, rice-

93 paddy complexes were considered spatially independent if further than $200 \mathrm{~m}$ apart, the

94 maximum daily dispersion distance for the species (Borzée et al. 2016a), or separated by

95 landscape barriers impermeable to treefrogs (Roh et al. 2014).

96 The Japanese Treefrog, D. japonicus is ubiquitously present on the wetlands of the

97 Korean Peninsula, and the two treefrog species are in sympatry at all sites. The advertisement

98 calls of D. japonicus and D. suweonensis are species specific (Jang et al. 2011; Park et al. 2013),

99 and we noted the presence or absence of $D$. suweonensis through acoustic monitoring. In calling

100 anurans, including Hylids, acoustic monitoring is known to be reliable to estimate population

101 size, and thus adequate to assess occurrence (Weir et al. 2005; Pellet et al. 2007; Dorcas et al.

102 2009; Petitot et al. 2014; Moreira et al. 2016). In a preliminary study, our aural survey protocol

103 with 5-min transects was accurate to estimate the occurrence of D. suweonensis (Borzée et al. 104 2017).

105 Transect surveys

106 We defined the general area for this study a priori, following the ecological requirements

107 of the species such as defined by Roh et al. (2014) and including all natural and man-made 108 wetlands west of $127.5^{\circ} \mathrm{E}$ and below $120 \mathrm{~m}$ above sea level. This pre-selection of potential 109 breeding sites through Google Earth Pro (Google Earth imagery, v7.1.2.2041, 2013) identified 110789 sites in 2014 (Fig. 1). A previous study for the occurrence of this species had drawn the 111 southern limit of the range around the Bay of Asan, below $37^{\circ} \mathrm{N}$ (Kim et al. 2012; Fig. 1). 
112 However, our surveys in 2014 demonstrated the southern limit of the range to be inaccurate

113 (Borzée et al. 2016c), and additional surveys were conducted further south in 2015 and 2016,

114 until reaching a point past where the species was no longer detected. In 2015, we surveyed 189

115 sites, composed of 90 new sites and 99 sites where the species was present in 2014. A single site

116 where the species was detected in 2014 could not be visited again due to its location within the

117 Civilian Control Zone (CCZ) adjacent to the border with the Democratic Republic of Korea and

118 the lack of permits for 2015 and 2016. In 2016, we surveyed a total of 122 sites (99 sites from

119 2014, 12 from 2015 and 11 new sites). All accessible sites where the species had been recorded in

1202014 were surveyed in 2015 and 2016, even if the species was not detected in 2015. All sites

121 where the species had been detected in 2015 were kept in the list of sites to survey in 2016. In

122 total, 890 sites were surveyed at least once over the three years of surveys.

123 Surveys were conducted between $5 \mathrm{pm}$ and $2 \mathrm{am}$, during the peak calling activity of the

124 species. After arrival at a survey site, five minutes were spent waiting quietly. For each site, aural

125 monitoring was conducted along a single transect along the centre of the rice-paddy complex. A

126 surveyor walked briskly at a maximum speed of circa $80 \mathrm{~m} / \mathrm{min}$ along the transect, noting the

127 presence or absence of $D$. suweonensis at the rice-paddy complex. Before conducting the project,

128 we had empirically measured the detection range for advertisement calls of $D$. suweonensis $(n=$

129 20), resulting in a $250 \pm 45 \mathrm{~m}$ range. The farthest rice paddies in rice-paddy complexes were

130 typically within this detection range.

At the end of each transect survey we recorded water $\mathrm{pH}$ and water conductivity $(\mu \mathrm{S})$ to

132 define the ecological preferences of $D$. suweonensis. We also estimated surface area and longest

133 straight line within sites to determine a sphericity ratio for the occurrence of the species. This is

134 important for determining the likelihood of a species' presence because a circular site will better

135 retain a species than a narrow and linear site. We then recorded the length of continuity with 
136 rivers and forests, defined as the continuous line between the edge of rice-paddy complexes and

137 the aforementioned landscape feature, and finally, we noted the presence of buildings and

138 greenhouses within the rice-paddy complexes. These variables were collected through the

139 drawing of polygons or visual inspection of sites in Google Earth Pro (Google Earth imagery,

140 v7.1.2.2041, 2016), at a $10 \mathrm{~m}$ resolution, on map dated from 2015 at the latest.

\section{Reclaimed lands and protected area}

142 To correlate the presence of the species with shifting landscape use, we recorded the 143 presence of the D. suweonensis at sites located on reclaimed lands. Here, reclaimed lands used to

144 be mudflats and sea beds, which have been converted into rice-paddy complexes. To record the 145 presence of reclaimed lands, we compared maps from 1950-51 drawn by the US Army (Center of 146 Military History 1990) downloaded in Google Earth and present satellite pictures from Google

147 Earth Pro (Google Earth imagery, 6.2.2.6613, 2016). The 1950-51 maps were selected due to 148 their precision. A land was considered reclaimed if it was not usable for breeding by $D$. 149 suweonensis in 1950-51, but converted into rice paddies before 2016.

150 We then compared the presence of protected areas and the localities where $D$. 151 suweonensis occurred. Data on protected areas were downloaded from the Protected Planet 152 database, set by the IUCN and UNEP-WCMC (2016). We subsequently noted the number of sites 153 within any protected area, as well as "sites that do not meet the standard definition of a protected 154 area but do achieve conservation in the long-term under national and international agreements" 155 (IUCN and UNEP-WCMC 2016).

\section{Origin of agricultural flood waters}

157 To analyse the impact of agricultural flood water on D. suweonensis distribution, we 158 asked rice farmers for the origin of the water used to flood their rice paddies. This survey was 
159 restricted to the general riverine basin surrounding the city of Iksan, south of the Geum River. To

160 be included in the analysis, the origin of the water for a rice paddy complex had to be confirmed

161 by at least two different farmers (Fig. 2). Data collection was limited to sites where surveys for

162 D. suweonensis were conducted. The area surveyed south of the city of Gunsan and the

163 Mankyeong River had to be excluded from the analysis due to lack of traceability of the origin of

164 agricultural flood water (Fig. 2).

\section{Data analysis and optimal conservation sites}

166 For subsequent analyses, we binary encoded the presence of the species, the presence of

167 greenhouses and the presence of permanent human infrastructures within the rice-paddy

168 complexes. We first determined the range of the species, based on presence data points (Fig. 1).

169 We defined the potential range of the species based on the non-interruption of landscape variables

170 that are within the range used by the species. We also delineated the ancestral range of the

171 species, defined as the potential range of the species before human development. Namely, a site

172 was considered potential for the species if $<120 \mathrm{~m}$ of altitude and within the same water basin as

173 a known population, excluding cities and urban area $>1 \mathrm{~km}^{2}$ (Fig. 1).

We then defined the overlap between species range and reclaimed area to estimate the

175 land use by the species, and calculated the overlap between species range and protected areas.

176 Descriptive statistics were used to characterise the impact of these landscape variables in both 177 cases.

We hypothesised the origin of the water to be important if linked to the Geum River. This 179 geographic area was chosen due to the clear segregation between areas flooded with water from 180 different origins. We indexed the occurrence of $D$. suweonensis at the sites surveyed in relation to 181 the binary encoding of the origin of flood water, from the Geum River. We subsequently assessed 182 whether distribution of $D$. suweonensis was random in relation to agricultural flood water. 
184 survey presence data, we calculated averages for water quality ( $\mathrm{pH}$ and conductivity) as proxies

185 for a larger set of values important for the species (Borzée et al. in review-b), the continuity with

186 rivers and forests, and the sphericity of sites. For sites surveyed over multiple years, the abiotic

187 variables used for the calculation of the species' preferences were restricted to the latest data

188 point. This choice to restrict the analysis to the survey presence data followed recent documented

189 local extinctions, and the potential for other undocumented local extinction due to water quality,

190 salinity, competition and land-use among others, and because these variables are important to

191 ecological preferences of species. All analyses were conducted with SPSS (v. 21.0, SPSS, Inc.,

192 Chicago, IL, USA), and maps were generated with ArcMap 9.3 (Environmental Systems

193 Resource Institute, Redlands, California, USA).

194 RESULTS

195 During the surveys conducted in 2014, only 358 sites out of the 789 sites pre-selected

196 were potentially habitable for the species as urban development and agricultural conversion

197 eliminated the 431 remaining sites. That is, these sites were beyond the ecological requirements

198 of the species as there was no standing water; instead the sites were mostly greenhouses,

199 apartment complexes or dry crops. Within the 358 habitable sites, we found calling Dryophytes

200 suweonensis at 100 sites, while the species was not detected at 258 sites. In 2015, calling $D$.

201 suweonensis were detected at 106 sites total, from 94 of the 100 sites where the species was

202 detected in 2014, and 12 of the new sites. In 2016, the species was detected at 109 sites total, 94

203 of the 2014 sites, 12 of the 2015 sites and three of the new sites. The 94 sites originating from the

2042014 dataset where the species was detected in 2015 and 2016 were the same. The species was

205 not detected at the five remaining sites where it had been found in 2014. The 12 sites where the

206 species was detected in 2015 were included in the surveys in 2016, and the species was again 
207 detected at all 12 sites. For all subsequent analyses, we assess the species to be present at the 114

208 sites where the species was detected at least once. This includes the 113 sites surveyed over three

209 years and the site behind the CCZ and these sites are distributed over circa $4300 \mathrm{~km}^{2}$ (Fig. 1).

210 However, this species is under significant threats of local extinction at the five sites where the

211 species was detected in 2014 only; a new motorway was built during the study period in the (Fig.

212 1, Bay of Asan).

213 Range, ancestral range and current optimal range

214 The southern boundary of D. suweonensis' distribution was extended $120 \mathrm{~km}$ southwards

215 from the previous assessment (Kim et al. 2012). The distribution of D. suweonensis ranges from

216 the southern banks of the Imjin River to the northern banks of the Mankyeong River, on a $220 \mathrm{~km}$

217 north-south transect. The range of the species spans $95 \mathrm{~km}$ longitudinally, with the westernmost

218 known population in Hongseong area and the easternmost in Wonju (Fig. 1).

The potential range of the species, defined as the area where ecological preferences of the

220 species are matched, is situated at the same latitude as the one where the species was detected,

221 but extends $25 \mathrm{~km}$ further west from the westernmost site where the species was detected,

222 towards the reclaimed Cheonsu bay. In addition, the corridor of low lands between Nonsan,

223 Gongju and Cheongju matches with the habitat required for the species, but no surveys were

224 conducted in that area, as primarily estimated too far and disconnected from the range of the

225 species to be a potential breeding area. When compared with the potential range of the species

226 before human development, referred here as ancestral range, the land surface area usable by the

227 species decreased by $729 \mathrm{~km}^{2}$ (Fig. 1).

\section{Overlap between reclaimed lands and protected area}

229 Out of the 114 sites where D. suweonensis was detected, a total of 30 sites were enlarged 230 and 15 sites were created through land reclamation. The remaining 69 sites were not impacted by 
231 land reclamation. When combining all sites impacted by land reclamation, they represent 39.47

$232 \%$ of the sites where D. suweonensis was present. When focusing on the overlap between the

233 occurrence of $D$. suweonensis and protected areas, only a single site was selected, South of

234 Pyeongtaek, protected under "Water Source Protection Area". In this protected site, only the

235 riverine system at the edge of the site is protected, putatively used by $D$. suweonensis for

236 hibernation and not for breeding.

\section{Origin of agricultural flood waters}

238 This analysis is based on a subset of sites in the southern distribution of the species (Fig.

239 2). A total of $53.3 \%$ of sites where $D$. suweonensis was present overlapped with agricultural

240 floods originating from the Geum River (Fig. 2), highlighting the non-random occurrence of the

241 species in this area. Few sites surveyed in the putatively suitable areas using water from the

242 Mankyeong River had D. suweonensis (1/15 sites; 7\%), while those in areas using water from the

243 Geum River had higher presence (8/17 sites; 49\%) and an even high proportion of sites showed

244 presence in the areas utilising underground water $(6 / 8$ sites; $75 \%)$. Autocorrelation of the origin

245 of flood water is likely, although of minor importance in this study and unlikely to impact the 246 result of the statistical analyses.

\section{Assessment of optimal conservation site}

The environmental variables for D. suweonensis (Table 1) showed an average $\mathrm{pH}$ of 8.32

249 and average conductivity of $792.19 \mu \mathrm{S}$. The average sphericity was 1.15 , meaning that sites were

250 more round than elongated in general. The majority of sites where D. suweonensis occurred had

251 permanent man-made infrastructures (52.9\%) and temporary structures (i.e. greenhouses, 68.9 
$252 \%)$ within the rice-paddy complexes.

253 Depiction of the sites adequate for the conservation of D. suweonensis (Fig. 3) was 254 supplemented by vegetation lists from Borzée and Jang (2015), and landscape information

255 matching the current habitat of D. suweonensis. Rice paddies are delimited by levees roughly 40 $256 \mathrm{~cm}$ wide and 20 to $60 \mathrm{~cm}$ high, covered with grasses, and used by treefrogs for basking, foraging, 257 and sheltering (Borzée et al. 2016a). The overhead view of the designed site highlights the need 258 for continuity with forests and rivers to match the preferences of the species (Fig. 3a), while the 259 lateral view (Fig. 3b) describes depth and vegetation characteristics required for the species.

DISCUSSION

261 This study highlights the importance of analysing data on the presence/absence and 262 habitat characteristics of species for their conservation. The known range of Dryophytes 263 suweonensis was doubled by the data collected over this three years study, highlighting the need

264 for a different approach to the selection of sites for the conservation of the species. These new 265 data show that the increase in known range is due to the inclusion of a large number of sites in 266 reclaimed area from post-war agricultural governmental development. The apparent expansion of 267 the species' known range is, however, countered by several potential local extirpations, such as 268 all the sites in the area of Suweon where the holotype for D. suweonensis was described 269 (Kuramoto 1980; Park et al. 2013).

270 The species still matches the criteria B1ab(i,ii,iii,iv) for listing as "endangered" under the criteria 271 of the International Union for Conservation of nature (IUCN) red list of endangered species. It

272 has an extent of occurrence $<5000 \mathrm{~km}^{2}$, a severely fragmented population with a continuing 273 observed decline for extent of occurrence, area of occupancy, quality of habitat and the number of 274 locations or subpopulations. At present, the protection of D. suweonensis is not ensured because 
275 no populations are located within a protected area. Only the edges of a single site are overlapping

276 with a protected area, south of Pyeongtaek. A single protected site is inadequate for the 277 conservation of an endangered species.

278 The description of the potential range for D. suweonensis shows that an area around 279 Cheongju may be adequate for the species to thrive. However, that area was not included in the 280 initial surveys, due to the lack of knowledge of such a potential wide range for the species.

281 Similarly, sites such as Baengnyeong or Seogmo Islands may be suitable but could not be 282 accessed due to their limited access to non-military personnel. Another potential significant range 283 increase would be within the Democratic People's Republic of Korea, as the species is known to 284 occur around Pyongyang (Chun et al. 2012).

285 Encroachment on the species' range by development (431 sites), such as at the sites 286 around the city of Suweon, has been partially counter-balanced by the land reclamation projects 287 for rice agriculture (15 sites) implemented at a very large scale in the Republic of Korea during 288 the second half of the last century. The presence of $D$. suweonensis on reclaimed land shows that 289 the species possesses the potential for dispersal despite a lower dispersal ability than the 290 sympatric Dryophytes japonicus (Borzée \& Jang 2016). This shift in range is thus linked to rice 291 cultivation and may have been an on-going process since early human agriculture circa 5000 292 years ago (Fuller et al. 2007; Fuller et al. 2008).

Furthermore, numerous D. suweonensis populations are isolated from each other, with 294 urbanization resulting in multiple landscape barriers within and among potential metapopulations.

295 This calls for a long-term study of population dynamics and network analysis for the species. We 296 would expect the population to be larger at reclaimed sites, due to lower levels of encroachment 297 and fragmentation. 
299 from the Geum River and underground water sources. However, the species was not detected at 300 sites flooded by water originating from the Mankyeong River. Thus, water originating from

301 underground water bodies and pumped to the surface for agricultural purposes may be adequate

302 for $D$. suweonensis. The areas flooded by river water may be the ones that were seasonally

303 flooded before landscape modifications by humans, and the absence of $D$. suweonensis at some

304 sites could relate to water quality. This idea is potentially supported by the absence of individuals

305 at the only site flooded by water originating from the Geum River south of the Mankyeong River.

306 However, as the water is brought by aerial channels, it is possible that some individuals $D$.

307 suweonensis will drift south to this area in the future and establish new colonies, or perhaps

308 hybridise with the D. japonicus present at the site (Borzée et al. 2015b).

309 Conservation of a species often requires the restoration of the species' habitat (Rannap et

310 al. 2009). The design of an optimal site for the protection of $D$. suweonensis highlights the need

311 for very large continuous flood plains. However, such large plains are becoming frequently

312 uncommon because of encroachment, and management plans have to be set before these sites

313 disappear. Furthermore, the presence of bullfrogs in the southern part of the range, may have a

314 known negative impact on the species (Borzée et al. in review-a), and demonstrates that the first

315 steps of conservation management plans for the species have to be conducted at any site where

316 the species would be protected/re-introduced. Finally, as the species is still present across a range

317 similar to its ancestral range, we do not recommend ex-situ conservation projects at this time, nor

318 translocations to new sites that would be outside of the ancestral range for the species.

We are extremely thankful to Mr. Yu Sang Hong for all the communication with the farmers, and 
321 without whom a large part of this manuscript would not exist.

\section{LITTERATURE CITED}

Abellán P, and Sánchez-Fernández D. 2015. A gap analysis comparing the effectiveness of Natura 2000 and national protected area networks in representing European amphibians and reptiles. Biodiversity and Conservation 24:1377-1390.

Borzée A, Ahn J, Kim S, Heo K, and Jang Y. 2015a. Seoul, Keep Your Paddies! Implications for the Conservation of Hylid Species. Animal Systematics, Evolution and Diversity 31:176-181.

Borzée A, Fong J, and Jang Y. 2015b. Broad introgression patterns between two Hylid species from the Korean Peninsula: the case of Hyla suweonensis and $\mathrm{H}$. japonica. The Korean Research Society of Herpetologists, Wonju, Republic of Korea.

Borzée A, and Jang Y. 2015. Description of a seminatural habitat of the endangered Suweon treefrog Hyla suweonensis. Animal Cells and Systems 19:1-5.

Borzée A, and Jang Y. 2016. Population persistence relates to behaviour and physiology in Korean Hylids. 16th congress of the International Society for Behavioral Ecology. International Society for Behavioral Ecology, Exeter, UK.

Borzée A, Kim JY, Cunha MAMd, Lee D, Sin E, Oh S, Yi Y, and Jang Y. 2016a. Temporal and spatial differentiation in microhabitat use: Implications for reproductive isolation and ecological niche specification. Integrative Zoology 11:375-387.

Borzée A, Kim JY, and Jang Y. 2016b. Calling site differentiation as a mechanism for reproductive isolation in two treefrog species. Scientific Reports 6.

Borzée A, Kosch TA, Kim M, and Jang Y. in review-a. Introduced bullfrogs are associated with increased Batrachochytrium dendrobatidis prevalence and reduced occurrence of Korean treefrogs. Biological Conservation.

Borzée A, Kyong CN, Kil HK, and Jang Y. in review-b. Impact of water quality on the occurrence of two endangered Korean anurans: Dryophytes suweonensis and Pelophylax chosenicus. Paddy and Water Environment.

Borzée A, Yu SH, and Jang Y. 2016c. Dryophytes suweonensis (Suweon Treefrog). Herpetological Review - Geographic Distribution 47:418.

Brooks TM, Bakarr MI, Boucher T, Da Fonseca GA, Hilton-Taylor C, Hoekstra JM, Moritz T, Olivieri S, Parrish J, and Pressey RL. 2004. Coverage provided by the global protected-area system: Is it enough? BioScience 54:1081-1091.

Center of Military History 1990. November 1950 - July 1951 - The United States Army in the Korean War. United States Army, Washington, D.C.

Chun S, Chung E, Voloshina I, Chong JR, Lee H, and Min M-S. 2012. Genetic Diversity of Korean Tree Frog (Hyla suweonensis and Hyla japonica): Assessed by Mitochondrial Cytochrome b Gene and Cytochrome Oxidase Subunit I Gene. Korean Journal of Herpetology 4:31-41.

Corsi F, De Leeuw J, and Skidmore A. 2000. Modeling species distribution with GIS. Research techniques in animal ecology:389-434.

Czech HA, and Parsons KC. 2002. Agricultural wetlands and waterbirds: a review. Waterbirds:5665.

Dorcas ME, Price SJ, Walls SC, and Barichivich WJ. 2009. Auditory monitoring of anuran 
populations. Amphibian ecology and conservation: a hand book of techniques. Oxford University Press, Oxford:281-298.

Duellman WE, Marion AB, and Hedges SB. 2016. Phylogenetics, classification, and biogeography of the treefrogs (Amphibia: Anura: Arboranae). Zootaxa 4104:1-109.

Dufresnes C, Borzée A, Horn A, Stöck M, Ostini M, Sermier R, Wassef J, Litvinchuk S, Kosch TA, Waldman B, Jang Y, Brelsford A, and Perrin N. 2015. Sex-chromosome homomorphy in Palearctic tree frogs proceeds from both turnovers and $X-Y$ recombination. Molecular Biology and Evolution msv113.

FAO. 2016. Food and Agriculture Organization Statistics. Food and Agriculture Organization of the United Nations.

Fuller DQ, Harvey E, and Qin L. 2007. Presumed domestication? Evidence for wild rice cultivation and domestication in the fifth millennium BC of the Lower Yangtze region. Antiquity 81:316-331.

Fuller DQ, Qin L, and Harvey E. 2008. Evidence for a late onset of agriculture in the Lower Yangtze region and challenges for an archaeobotany of rice. Pages 40-83 in R. M. In: Blench R, Lin $\mathrm{M}$ et al. (eds) Human Migrations in Continental East Asia and Taiwan, editor. Human migrations in continental East Asia and Taiwan: Genetic, Linguistic and Archaeological Evidence. Taylor \& Francis, London.

Huxley C. 2013. CITES: the vision. Page 3. Endangered Species Threatened Convention: The Past, Present and Future of CITES, the Convention on International Trade in Endangered Species of Wild Fauna and Flora. Routledge.

IUCN. 2016. The IUCN Red List of Threatened Species. <http://www.iucnredlist.org> Version 2016.2.

IUCN and UNEP-WCMC. 2016. The World Database on Protected Areas (WDPA), Cambridge, UK: UNEP-WCMC.

Jang Y, Hahm EH, Lee H-J, Park S, Won Y-J, and Choe JC. 2011. Geographic variation in advertisement calls in a tree frog species: gene flow and selection hypotheses. PLoS One 6:e23297.

Juliano BO 1993. Rice in human nutrition. and International Rice Research Institute.

Kim I-H, Son S-H, Kang S-W, and Kim J-B. 2012. Distribution and Habitat Characteristics of the Endangered Suweon-Tree Frog (Hyla suweonensis). Korean Journal of Herpetology 4:1522.

Kuramoto M. 1980. Mating calls of treefrogs (genus Hyla) in the far east, with description of a new species from Korea. Copeia 1:100-108.

Mace GM, and Lande R. 1991. Assessing extinction threats: toward a reevaluation of IUCN threatened species categories. Conservation Biology 5:148-157.

Machado IF, and Maltchik L. 2010. Can management practices in rice fields contribute to amphibian conservation in southern Brazilian wetlands? Aquatic Conservation: Marine and Freshwater Ecosystems:n/a-n/a.

Manne LL, and Pimm SL. 2001. Beyond eight forms of rarity: which species are threatened and which will be next? Animal Conservation 4:221-229.

Miller RR, Williams JD, and Williams JE. 1989. Extinctions of North American fishes during the past century. Fisheries 14:22-38.

Mizsei E, Üveges B, Vági B, Szabolcs M, Lengyel S, Pfliegler WP, Nagy ZT, and Tóth JP. 2016. Species distribution modelling leads to the discovery of new populations of one of the least known European snakes, Vipera ursinii graeca, in Albania. Amphibia-Reptilia 37:55- 
408

409

410

411

412

413

414

415

416

417

418

419

420

421

422

423

424

425

426

427

428

429

430

431

432

433

434

435

436

437

68.

Moreira LF, Moura RG, and Maltchik L. 2016. Stop and ask for directions: factors affecting anuran detection and occupancy in Pampa farmland ponds. Ecological Research 31:65-74.

Park D, Min M-S, Lasater K, Song J-Y, Suh J-H, Son S-H, and Kaplan R 2014. Conservation of amphibians in South Korea. I. Das, M. Wilkinson, and H. Heatwole (eds.).

Park S, Jeong G, and Jang Y. 2013. No reproductive character displacement in male advertisement signals of Hyla japonica in relation to the sympatric $H$. suweonensis. Behavioral Ecology and Sociobiology 67:1345-1355.

Pellet J, Helfer V, and Yannic G. 2007. Estimating population size in the European tree frog (Hyla arborea) using individual recognition and chorus counts. Amphibia-Reptilia 28:287-294.

Petitot M, Manceau N, Geniez P, and Besnard A. 2014. Optimizing occupancy surveys by maximizing detection probability: application to amphibian monitoring in the Mediterranean region. Ecology and evolution 4:3538-3549.

Pimm SL, Jenkins CN, Abell R, Brooks TM, Gittleman JL, Joppa LN, Raven PH, Roberts CM, and Sexton JO. 2014. The biodiversity of species and their rates of extinction, distribution, and protection. Science 344:1246752.

Purvis A, Gittleman JL, Cowlishaw G, and Mace GM. 2000. Predicting extinction risk in declining species. Proceedings of the Royal Society of London B: Biological Sciences 267:19471952.

Rannap R, Lohmus A, and Briggs L. 2009. Restoring ponds for amphibians: a success story. Hydrobiologia 634:87-95.

Roh G, Borzée A, and Jang Y. 2014. Spatiotemporal distributions and habitat characteristics of the endangered treefrog, Hyla suweonensis, in relation to sympatric $H$. japonica. Ecological Informatics 24:78-84.

Stuart SN, Chanson JS, Cox NA, Young BE, Rodrigues AS, Fischman DL, and Waller RW. 2004. Status and trends of amphibian declines and extinctions worldwide. Science 306:17831786.

Weir LA, Royle JA, Nanjappa P, and Jung RE. 2005. Modeling Anuran Detection and Site Occupancy on North American Amphibian Monitoring Program (NAAMP) Routes in Maryland. Journal of Herpetology 39:627-639. 
438 TABLES

439 Table 1:

440 Descriptive statistics for abiotic variables of interest collected from all sites where Dryophytes

441 suweonensis was present.

\begin{tabular}{lccccc}
\hline \hline & $\mathrm{N}$ & Min & Max & Mean & Std \\
\hline Water $\mathrm{pH}$ & 114 & 7.20 & 10.20 & 8.32 & 0.32 \\
Water conductivity $(\mu \mathrm{S})$ & 114 & 83.50 & 5720.00 & 792.19 & 740.47 \\
Surface area $\left(\mathrm{m}^{2}\right)$ & 114 & 0.31 & 26.09 & 4.78 & 4.36 \\
Max. length $(\mathrm{km})$ & 114 & 1.10 & 301.00 & 6.30 & 27.89 \\
Continuity with forests $(\mathrm{km})$ & 114 & 0.00 & 14.10 & 3.87 & 2.83 \\
Continuity with rivers $(\mathrm{km})$ & 114 & 0.00 & 9.20 & 1.17 & 1.79 \\
Sphericity & 114 & 0.01 & 2.87 & 1.15 & 0.65 \\
\hline \hline
\end{tabular}


443 FIGURES

444 Figure 1:

445 Summary of the 890 sites surveyed at least once over the three years of surveys. Dryophytes 446 suweonensis was detected at least once at 114 sites, and 421 sites were too excessively urbanised

447 for the species to occur. Here, potential current range is defined as the range where the species 448 could currently occur, while the potential ancestral range is the range where the species could 449 have occurred before urban development. Pyeongtaek is the area where the only protected area 450 with D. suweonensis is found. 


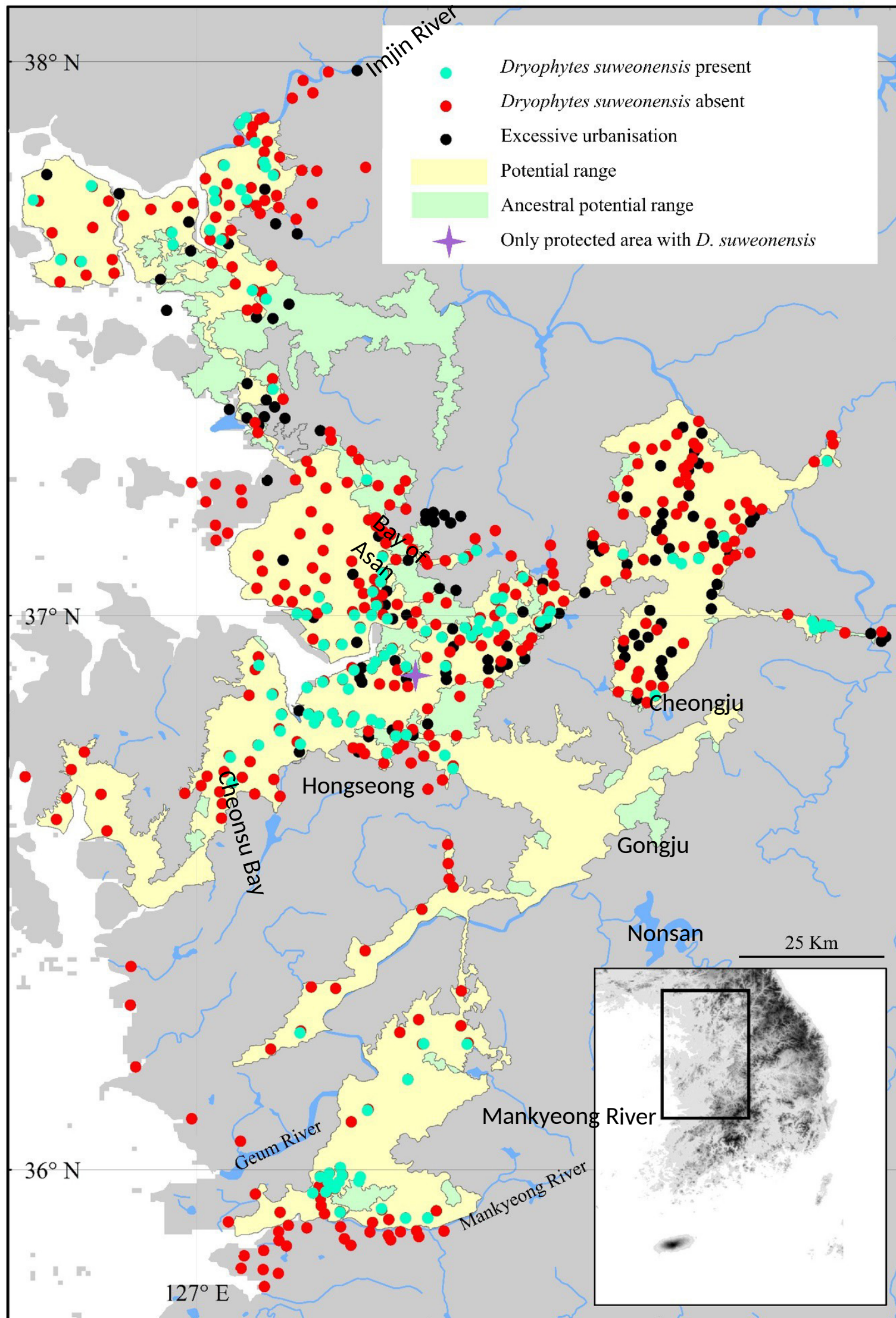

Wonj 


\section{Figure 2:}

452 Relationship between flood water origin and species presence. Most of the flood water matching 453 with the occurrence of Dryophytes suweonensis originated from the Geum River (53.3\%), 454 followed by underground water (40\%), while the remaining $6.7 \%$ of sites were flooded by the 455 Mankyeong river. This analysis is restricted to the area shown on the map. 


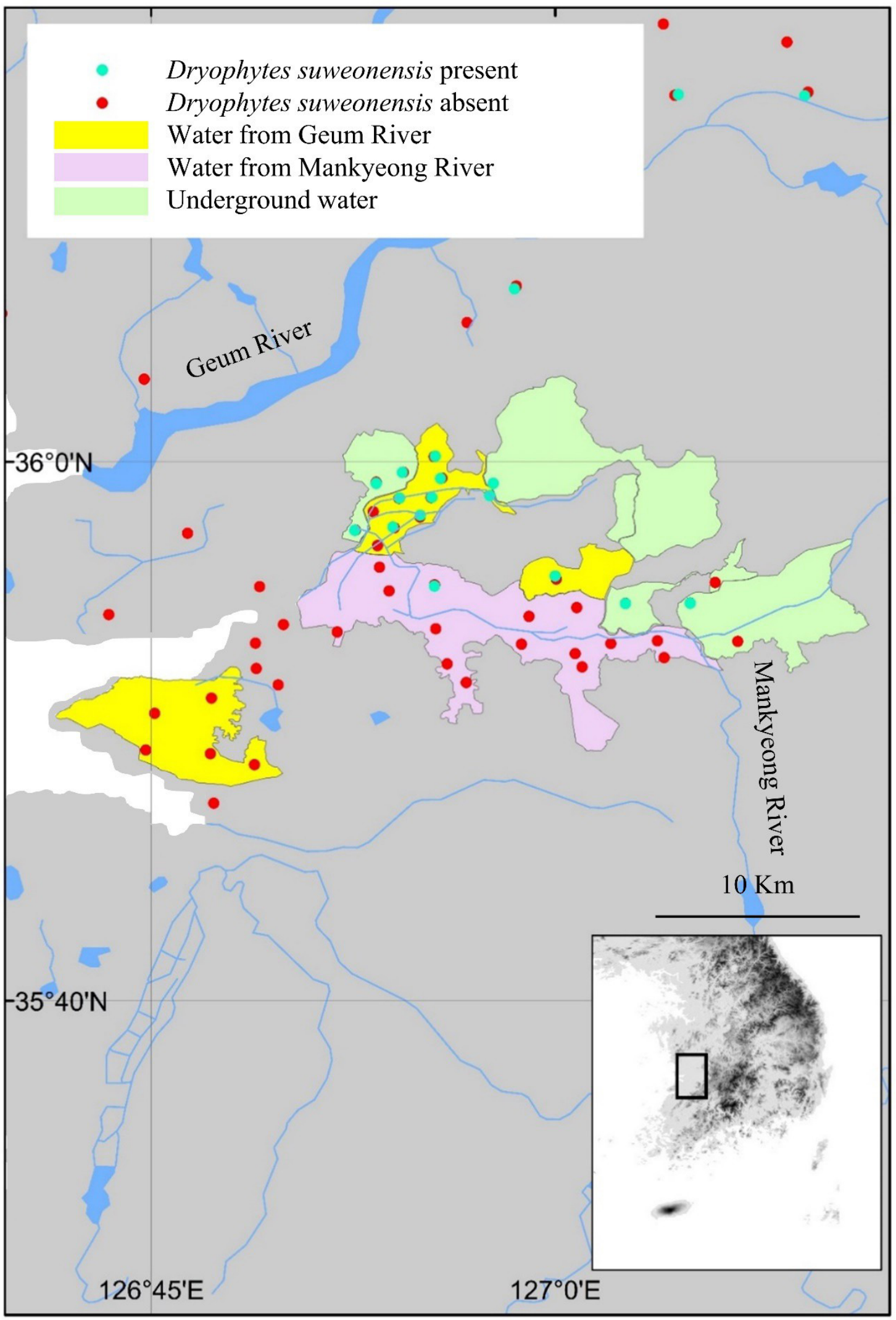


457 Figure 3:

458 (A) Overhead view of the site optimally designed to follow ecological preferences demonstrated 459 by Dryophytes suweonensis. The cut AA' is reported in Fig. 3b. The figure is not to scale. Water 460 depths indicated are matching with the average depth of rice paddies, and therefore acceptable if 461 not optimal for the species, and vegetation data is extracted from Borzée and Jang (2015). (B) 462 Lateral view of the site optimally designed to follow ecological preferences demonstrated by 463 Dryophytes suweonensis. Water level (WL) originates from the only known natural site with 464 Dryophytes suweonensis (Borzée and Jang 2015). 

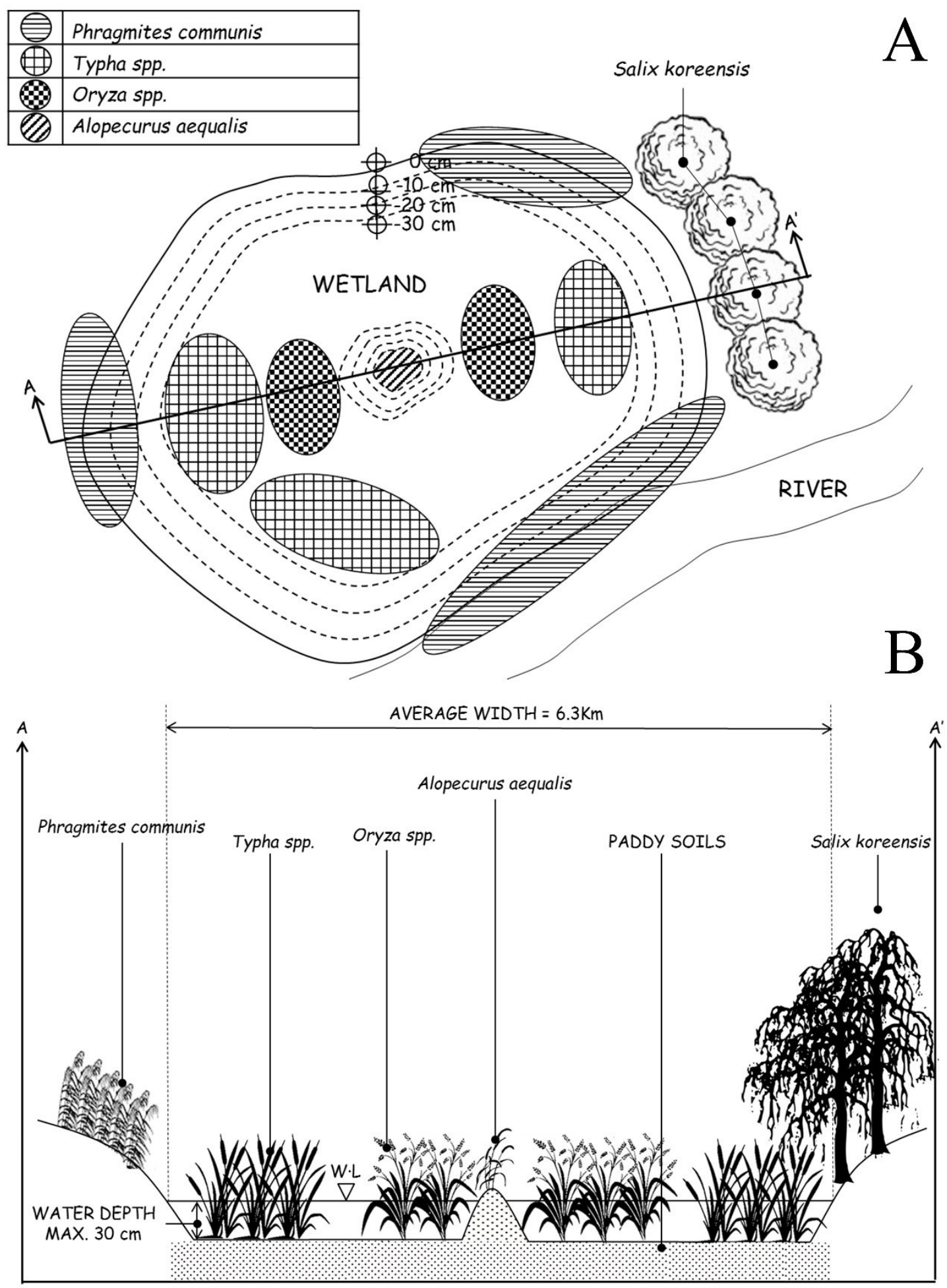